\title{
Tratamientos hormonales aplicados posterior a la IATF sobre la tasa de preñez y la viabilidad embrionaria en vacas de alta producción en estrés térmico
}

\author{
Hormonal treatments applied after the FTAI on pregnancy rate and embryo \\ viability in high-producing dairy cows under thermal stress \\ Yusep Gómez Marín ${ }^{1,4}$, José Goicochea Vargas', Wilson Rondón Jorge ${ }^{1}$, Fidel \\ Acosta Pachorro ${ }^{1}$, Magaly Montalvo Martin ${ }^{1}$, Marce Pérez Saavedra ${ }^{1}$, Carlos \\ Ballarte Zevallos ${ }^{1}$, Mauricio Silva Jiménez ${ }^{3}$, Marcelo Ratto Fuster ${ }^{2}$
}

\section{Resumen}

\begin{abstract}
El objetivo del estudio fue evaluar el efecto de GnRH, hCG y eCG aplicados 14 días después de la inseminación artificial a tiempo (IATF) fijo sobre la tasa de preñez y sobrevivencia embrionaria y fetal en vacas sometidas a estrés calórico. Las vacas $(n=234)$ fueron sincronizadas e inseminadas y recibieron diferentes dosis hormonales vía i.m. 14 días después: a) Grupo control: $2 \mathrm{ml}$ de suero fisiológico (n=56), b) Grupo GnRH: $100 \mu \mathrm{g}$ de GnRH (n=58), Grupo c) hCG: 1500 UI de hCG (n=60), y d) Grupo eCG: 400 UI de eCG $(\mathrm{n}=60)$. El diagnóstico de gestación y la evaluación del número de cuerpos lúteos (CL) se determinó mediante ultrasonografía a los días 34, 45 y 60 de la IA. Las vacas tratadas con GnRH presentaron una mayor tasa de preñez $(\mathrm{p}<0.05)$ y sin diferencias significativas entre los demás grupos. La sobrevivencia embrionaria y fetal entre los días 45 y 60 fue constante en todos los grupos, pero ocurrió una muerte fetal en el grupo eCG. No hubo diferencia significativa en la proporción de cuerpos lúteos accesorios entre los grupos tratados y el grupo control a los 60 días de gestación. Se concluye que la administración de GnRH 14 días pos-IATF mejora la tasa de preñez en vacas lecheras sometidas a estrés calórico.
\end{abstract}

Palabras clave: vacas de alta producción; GnRH; tasa de preñez; progesterona; cuerpo lúteo

\footnotetext{
${ }^{1}$ Laboratorio de Cirugía y Biotecnología Reproductiva, Facultad de Medicina Veterinaria y Zootecnia, UNHEVAL, Huánuco-Perú

${ }^{2}$ Instituto de Ciencia Animal, Universidad Austral de Chile

${ }^{3}$ Laboratorio de Reproducción Animal, Universidad Católica de Temuco

${ }^{4}$ E-mail:yusep111@hotmail.com
}

Recibido: 5 de marzo de 2019

Aceptado para publicación: 30 de octubre de 2019 
The aim of this study was to evaluate the effect of GnRH, hCG and eCG applied 14 days after fixed time artificial insemination (FTAI) on the rate of pregnancy and embryonic and foetal survival in cows under heat stress. The cows $(n=234)$ were synchronized, inseminated and received 14 days later via i.m. the hormonal application: a) Control group: $2 \mathrm{ml}$ of saline solution ( $\mathrm{n}=56), \mathrm{b}) \mathrm{GnRH}$ group: $100 \mu \mathrm{g}$ of $\mathrm{GnRH}(\mathrm{n}=58)$, Group c) hCG: $1500 \mathrm{IU}$ of hCG ( $\mathrm{n}=60)$, and d) ECG Group: $400 \mathrm{IU}$ of eCG $(\mathrm{n}=60)$. The diagnosis of pregnancy and the evaluation of the number of corpora lutea (CL) was determined by ultrasonography on days 34, 45 and 60 of the AI. Cows treated with GnRH had a higher pregnancy rate $(\mathrm{p}<0.05)$ and no significant differences were found between the other groups. Embryonic and foetal survival between days 45 and 60 was constant in all groups, but one foetal death occurred in the eCG group. There were no significant differences in the proportion of accessory CL between the treated groups and the control group at 60 days of gestation. It is concluded that the administration of GnRH 14 days post-FTAI improves the pregnancy rate in dairy cows under caloric stress.

Key words: high-producing cows; GnRH; pregnancy rate; progesterone; corpus luteum

\section{INTRODUCCIÓN}

Uno de los grandes problemas en los sistemas de producción lechero es la elevada tasa de mortalidad embrionaria (Thatcher $e t$ al., 1994; Vanroose et al., 2000; Sreenan et al., 2001). Según Wathes (1992), la mayoría de estas pérdidas embrionarias se producen en los primeros días de la fecundación y durante la implantación del embrión. Se considera que una fecundación fértil $(80-100 \%)$ por monta natural es seguida por una tasa de concepción del $70 \%$, de allí que pérdidas entre el 10 y el $30 \%$ pueden deberse a problemas relacionados con el reconocimiento materno y factores genéticos, de manejo, estrés y de salud entre otros (Diskin y Morris, 2008).

En el tema de mortalidad embrionaria se deben diferenciar dos grandes momentos en el desarrollo del concepto: la etapa previa al reconocimiento materno de la preñez (primeros 14 días) y la etapa del desarrollo embrionario tardío que corresponden a la etapa después del reconocimiento materno de la gestación (BonDurant, 2007). En este segundo periodo no solamente se da el proceso de organogénesis, sino que también se inicia la formación de la placenta, la cual culmina hacia el día 90 de gestación, de allí que cualquier alteración entre la fertilización y los 90 días de desarrollo embrionario-fetal tienden a ser letales (BonDurant, 2007; Diskin y Morris, 2008).

Bartolomé et al. (2012) indica que la aplicación de la hormona gonadotrofina coriónica equina (eCG) al día 22 después de la Inseminación Artificial a Tiempo Fijo (IATF) incrementa el número de vacas preñadas con baja condición corporal, y que la aplicación de la hormona gonadotrofina corionica humana (hCG) al día 29 de la gestación reducía las perdidas embrionarias e incrementaba el número de cuerpos lúteos (CL) accesorios, pero el uso de hCG al día 29 de la gestación sin el uso de eCG al día 22 reducía la fertilidad.

Es posible que el manejo farmacológico pos-inseminación tenga un efecto positivo, no solamente sobre la función luteal, sino que también con la remodelación del endometrio para mejorar el reconocimiento materno y el inicio de la implantación. Por tal motivo, el 
presente trabajo estudió el efecto de la administración de la hormona liberadora de gonadotrofinas ( $\mathrm{GnRH})$, hCG y eCG al día 14 pos-inseminación evaluando las tasas de preñez y mortalidad embrionaria en ganado lechero de alta producción.

\section{Materiales y Métodos}

El presente estudio se llevó a cabo en la Lechería ANCALI de Los Ángeles, VIII región, Chile, durante los meses de diciembre de 2012 hasta marzo de 2013 ( $\mathrm{T}^{\circ}$ máxima y humedad relativa promedio del periodo: $27.7 \pm 3.6{ }^{\circ} \mathrm{C}$ y $53.4 \pm 13.4 \%$ ), en colaboración con el Laboratorio Pfizer y el Instituto de Ciencia Animal (Laboratorio de Reproducción Animal) de la Facultad de Ciencias Veterinarias de la Universidad Austral de Chile.

Se utilizaron 234 vacas lecheras entre 1 a 6 partos, con un rango de peso vivo desde los 530 a $640 \mathrm{~kg}$, promedio de días posparto de $135.7 \pm 89$, número de lactancias $1.9 \pm$ 1.0 , condición corporal de $3.28 \pm 0.23$ y producción diaria de leche de $45 \pm 4.0$ L. Los animales fueron manejados en un sistema free stall sometidas a tres ordeños diarios, alimentados con una dieta basada en ensilaje de maíz y alfalfa recibiendo una ración TMR (Ración Total Mezclada) más agua ad libitum. A todos los animales se les realizó un examen reproductivo mediante el uso de un espéculo más ecografía transrectal (Well WED-180 con un transductor lineal de $6.5 \mathrm{Mhz}$ ) para excluir vacas con patologías ováricas o uterinas.

\section{Diseño Experimental}

Las vacas $(\mathrm{n}=234)$ fueron sincronizadas mediante un protocolo estándar de sincronización de celo-ovulación para IATF. Al inicio del tratamiento (día -10) recibieron $2 \mathrm{mg}$ de benzoato de estradiol (Syntex) i.m. y un dispositivo intravaginal con $1.39 \mathrm{~g}$ de progesterona (CIDR ${ }^{\circledR}$, Pfizer). Los dispositivos fueron retirados 8 días después (día -2) y se les aplicó vía i.m. $25 \mathrm{mg}$ de Trometamina de Dinoprost-PGF ${ }_{2 \dot{a}}$ (Lutalyse ${ }^{\circledR}$, Pfizer), 400 UI de eCG (Novormon ${ }^{\circledR}$, Syntex) y $1 \mathrm{mg}$ de cipionato de estradiol (ECP $®$, Pfizer). La inseminación artificial a tiempo fijo (IATF) fue realizada a las $52-56 \mathrm{~h}$ posterior al retiro del CIDR (día 0).

Al día 14 posterior a la IATF, los animales fueron asignados al azar a cuatro grupos experimentales para recibir una administración intramuscular de: A: Suero salino $(\mathrm{n}=56)$; B: $100 \mu \mathrm{g}$ de un agonista de $\mathrm{GnRH}$, acetato de gonadorelina (Fertiline, $\mathrm{n}=58$ ); C: 1500 UI de hCG (n=60); D: 400 UI de eCG (n=60). Diez vacas de cada grupo fueron ecografiadas los días 14,16 y 22 pos-IATF para detectar cambios en la estructura luteal y la posible presencia de CL accesorios; además se les tomó una muestra de sangre los días 14, 16, 18,20 y 22 para determinar la concentración plasmática de progesterona. El diagnóstico de gestación (DX) y seguimiento de la preñez fue realizado en todos los animales mediante examen ecográfico los días 34,42 y 60 pos-IATF.

\section{Progesterona Plasmática}

Las muestras de sangre $(5-7 \mathrm{ml})$ se tomaron de la vena coccígea y colectadas en tubos con $100 \mu l$ de heparina. Las muestras se almacenaron en hielo hasta la centrifugación que se realizó dentro de una hora a $2000 \mathrm{rpm}$ durante 15 minutos. El plasma se separó y congeló a $-20{ }^{\circ} \mathrm{C}$ hasta la determinación de la concentración de progesterona usando un kit comercial de radioinmunoanálisis (kit PROG-RIA-CT, DIA Source ImmunoAssays, Belgium). El coeficiente de variación intra-ensayo fue de 4.1 y $3.3 \%$ para baja y alta concentración plasmática respectivamente $(1.27 \pm 0.05 \mathrm{y}$ $4.08 \pm 3.3 \mathrm{ng} / \mathrm{ml}$, respectivamente). El coeficiente de variación inter-ensayo fue de 8.6 y $6.5 \%$ para baja y alta concentración plasmática, respectivamente $(1.17 \pm 0.10 \mathrm{y}$ $3.93 \pm 0.26 \mathrm{ng} / \mathrm{ml})$. La sensibilidad del ensayo fue de $0.05 \mathrm{ng} / \mathrm{ml}$. 
Cuadro 1. Tasa de preñez (\%) y proporción de vacas con presencia de cuerpo lúteo accesorio (CLa) en hembras tratadas con $\mathrm{GnRH}, \mathrm{hCG}$ y eCG 14 días post-IATF

\begin{tabular}{lllll}
\hline & $\begin{array}{l}\text { Control } \\
(\mathrm{n}=56)\end{array}$ & $\begin{array}{l}\text { GnRH } \\
(\mathrm{n}=58)\end{array}$ & $\begin{array}{l}\mathrm{hCG} \\
(\mathrm{n}=60)\end{array}$ & $\begin{array}{l}\mathrm{eCG} \\
(\mathrm{n}=60)\end{array}$ \\
\hline Preñez día 34 & $8.9^{\mathrm{a}}$ & $33.0^{\mathrm{b}}$ & $8.1^{\mathrm{a}}$ & $10.0^{\mathrm{a}}$ \\
Preñez día 45 & $8.9^{\mathrm{a}}$ & $33.0^{\mathrm{b}}$ & $8.1^{\mathrm{a}}$ & $10.0^{\mathrm{a}}$ \\
Preñez día 60 & $8.9^{\mathrm{a}}$ & $33.0^{\mathrm{b}}$ & $8.1^{\mathrm{a}}$ & $8.1^{\mathrm{a}}$ \\
\hline
\end{tabular}

$a, b$ Letras diferentes dentro de una misma fila difieren significativamente $(p<0.05)$
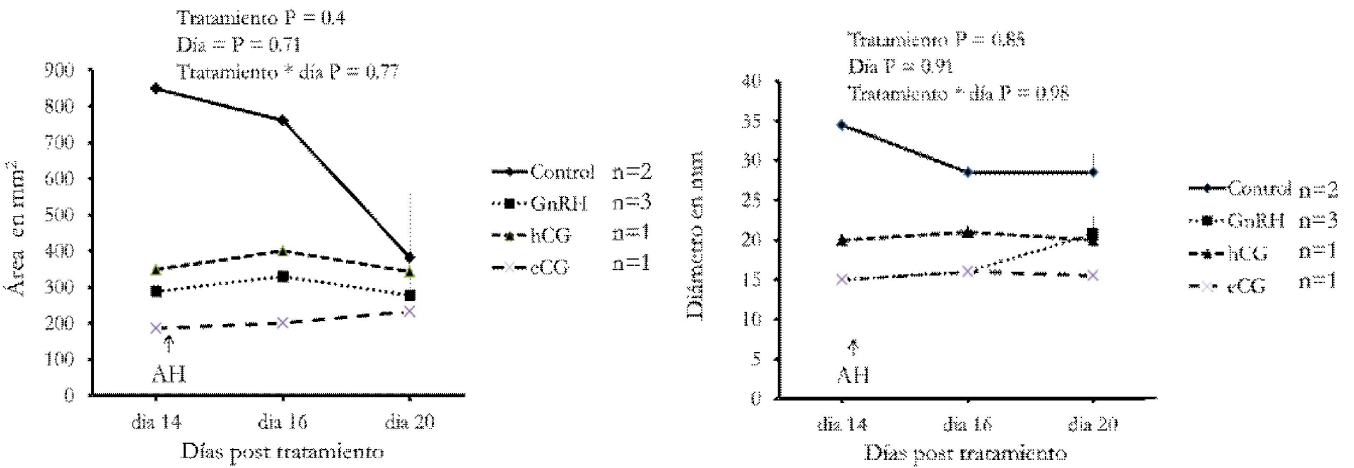

Figura 1. Área del cuerpo lúteo (izquierda) y del diámetro del cuerpo lúteo (derecha) en vacas preñadas tratadas con $\mathrm{GnRH}$, hCG y eCG al día 14 po-IATF

\section{Análisis Estadístico}

La tasa de preñez fue comparada entre los grupos mediante la prueba de Chi cuadrado. Las concentraciones plasmáticas de progesterona fueron analizadas mediante análisis de varianza para muestras repetidas mediante MIXED modelo. La comparación de las medias se hizo con las pruebas de Tukey, Duncan y Bonferoni en el programa SAS 1996.

\section{Resultados}

\section{Tasa de Preñez}

La tasa de preñez fue significativamente mayor en las vacas tratadas con-
GnRH $(\mathrm{p}<0.05)$ en todos los periodos de evaluación, mientras que fue menor y constante para los grupos Control y hCG. En el grupo eCG se observó una pérdida fetal entre el día 45 y 60 de la gestación, representando el 3\% del total de vacas preñadas del estudio (Cuadro 1). En el grupo tratado con hCG se observó una mayor, pero no significativa proporción de vacas preñadas con CLa.

El área total de tejido luteal y el diámetro del CL permanecieron constantes en las vacas preñadas (Figura 1); sin embargo, en las vacas que no llegaron a preñar, el área total de tejido luteal y el diámetro del CL fueron estadísticamente mayores $(\mathrm{p}<0.02)$ en los grupos Control, GnRH y hCG con respecto al grupo eCG al día 16 pos-tratamiento (Figura 2). 

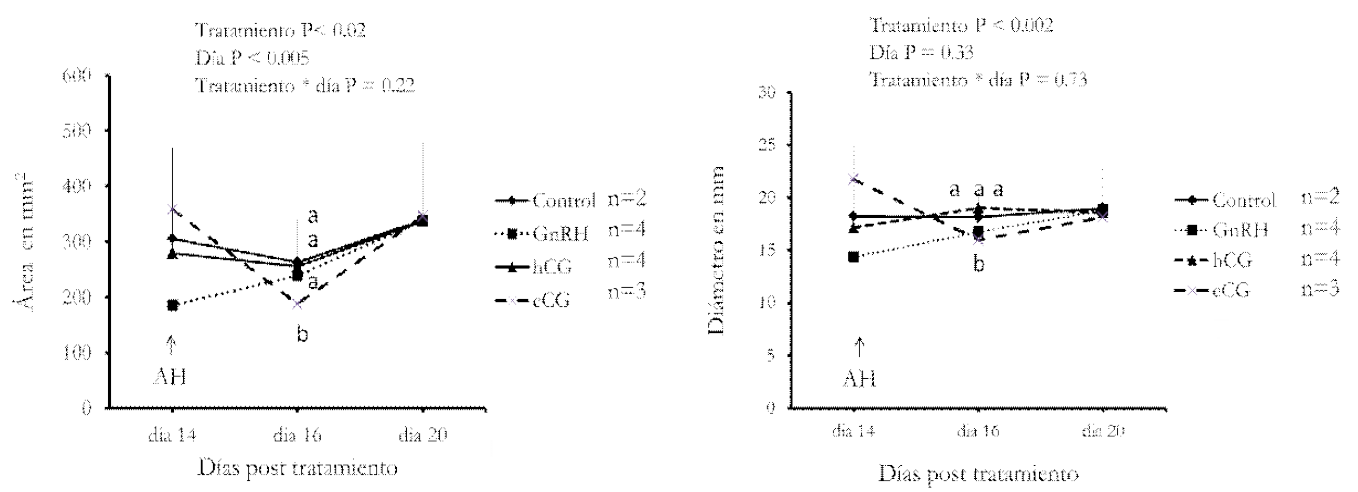

Figura 2. Área del cuerpo lúteo (izquierda) y del diámetro del cuerpo lúteo (derecha) en vacas vacías tratadas con GnRH, hCG y eCG al día 14 po-IATF. ${ }^{\text {a,b }}$ Letras diferentes indican diferencia significativa entre grupos $(p<0.05)$
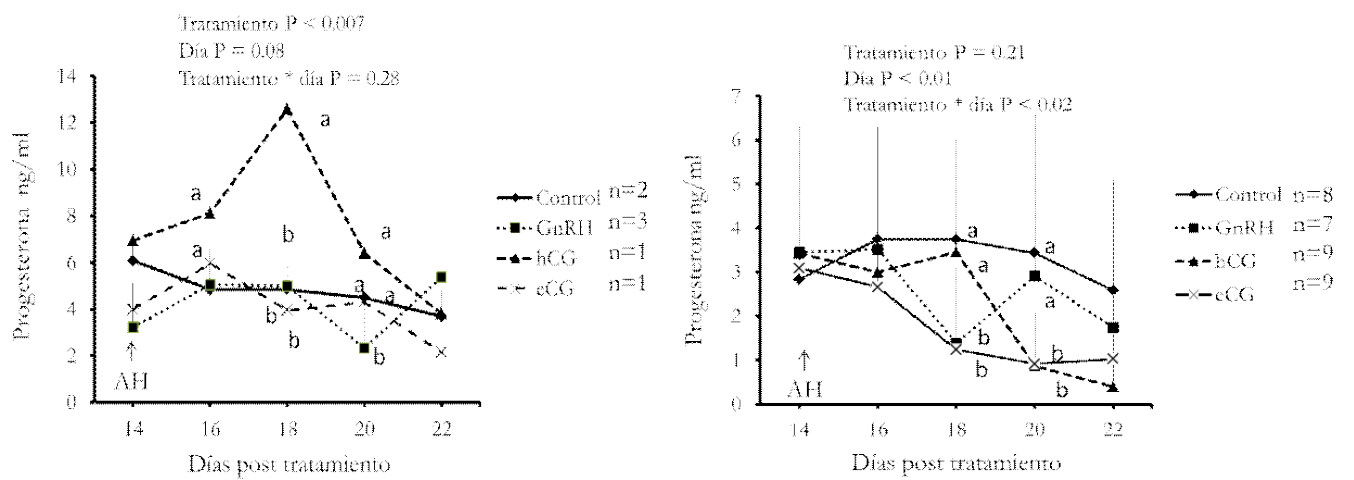

Figura 3. Concentración de progesterona en vacas preñadas (izquierda) y vacías (derecha) tratadas con $\mathrm{GnRH}$, hCG y eCG al día 14 pos-IATF. a,b Letras diferentes indican diferencia entre grupos $(p<0.05)$

La concentración de progesterona en vacas preñadas fue significativamente mayor en las vacas tratadas con hCG $(\mathrm{p}<0.007)$ desde el día 16 hasta el día 20 pos-tratamiento (Figura 3). Así mismo, la concentración de progesterona plasmática en las vacas vacías fue significativamente mayor en el grupo Control y en las tratadas con hCG $(\mathrm{p}<0.01)$ los días 18 y 20 pos-tratamiento (Figura 3 ).

\section{Discusión}

En el presente estudio se demostró que la GnRH tuvo un efecto positivo en la eficiencia reproductiva del ganado lechero, al aumentar la tasa de preñez y mantener la sobrevivencia embrionaria fetal en el grupo tratado, luego de su aplicación 14 días posteriores a la inseminación artificial en compa- 
ración al grupo control y los grupos tratados con eCG y hCG. Se debe destacar, asimismo, que las tasas de preñez obtenidas en estos últimos grupos fueron bajas. Contrastando datos reportados por otros autores, Bartolome et al. (2012) obtierron 50\% de preñez al administrar eCG el día 22 de la IA en comparación con el 33\% del grupo control, mientras que Narváez (2010) obtuvo $50.6 \%$ con eCG entre los días 16 y 22 y $44.9 \%$ para el grupo control. Con el uso de hCG, Rajamahendran y Sianangama (1992) reportan tasas de 50, 78 y $44 \%$ de preñez luego de la administración los días 0,7 y 14 de la IA, respectivamente, mientras que Santos et al. (2001) administrando hCG 5 días de la IA encontraron tasas de preñez en los grupos tratados y control de 46 vs $39 \%$ al día 28,40 vs $36 \%$ al día 45 y 38 vs $32 \%$ al día 90 de la IA. Por otro lado, Rendel (1997) reportaron tasas de preñez en Chile de $52-55 \%$ para vacas tratadas con $\mathrm{GnRH}$ al momento de la IA y 7 días después.

En la evaluación de los resultados obtenidos se debe considerar que la fase experimental fue llevada a cabo entre diciembre y marzo, con temperatura ambiental de 27.7 $\pm 3.4{ }^{\circ} \mathrm{C}$ y humedad de $52 \pm 13 \%$, mientras que el rango óptimo de temperatura para el ganado bovino lechero se encuentra entre 13 y $18{ }^{\circ} \mathrm{C}$, con temperatura límite entre $21.1 \mathrm{y}$ $32.2^{\circ} \mathrm{C}$ (López et al., 2003). El aumento de la temperatura ambiental tiene un impacto nocivo en la duración del estro, en el crecimiento y desarrollo folicular y en el desarro1lo embrionario (De Rensis y Scaramuzzi, 2003; Jordan, 2003). En un estudio de 10 años em España, López-Gatius (2003) reportó tasa de preñez promedio para los animales inseminados en el periodo de verano de $27.4 \%$, mientras que en otros periodos del año fue de $44.4 \%$.

La tasa de preñez entre el grupo control y el grupo tratado con eCG fue similar, lo cual no concuerda con resultados obtenidos por otros autores, quienes reportaron frecuencias de preñez mayores con el uso de eCG (Núñez, 2011; Bartolome et al., 2012).
El grupo tratado con GnRH presentó una tasa de preñez significativamente mayor que los grupos tratados con eCG, hCG y del control, sugiriendo que esta hormona podría tener un mayor efecto sobre la fertilidad de vacas lecheras que experimentan estrés térmico en comparación a los otros preparados hormonales. Los resultados son coincidentes con los trabajos de Willard et al. (2003) y Lopez-Gatius et al. (2006), quienes aplicaron $\mathrm{GnRH}$ en días posteriores a la IA a vacas lecheras bajo condiciones estrés por calor.

La mortalidad embrionaria en el ganado bovino ha sido estimada entre 20 y $40 \%$ (Vanroose et al., 2000; Sreenan et al., 2001), e incluso menor (Santos et al., 2004). En el presente estudio solo se pudo detectar una pérdida fetal en el grupo tratado con eCG, pero se debe tomar en consideración que la metodología empleada no permitió detectar pérdidas embrionarias antes de los 20-22 días de la IA. Al igual que en este estudio, Núñez (2011) reportó pérdidas de gestación en vacas de carne al día 60, luego de la aplicación de eCG 14 días pos-IA; así mismo, Bartolome et al. (2012) reportaron pérdidas (3/40) en ganado lechero tratado con eCG a los 22 días pos-IA, pero menores que en el grupo control $(6 / 28)$.

No se encontraron diferencias en el número de $\mathrm{CL}$ accesorios inducidos por los tratamientos hormonales en el presente trabajo. No obstante, Bartolome et al. (2006) observaron un mayor número de CL accesorios en vacas con implantes de Deslorelina al día 45 de gestación comparado con el grupo control (45.4 vs $10.1 \%$ ), y sin que llegasen a presentar pérdidas fetales. Un estudio en el país reportó CL accesorios en el 78.7\% de vacas tratadas con Buserelina al día 7 posIA (Rendel 1997), mientras que GarciaIspierto y López-Gatius (2012) encontraron nuevas estructuras lúteas en el $60 \%$ de las vacas luego de la administración de GnRH 5 días pos-IA. En diferentes estudios, la administración de hCG y GnRH en distintos momentos pos-IA aumentó el número de $\mathrm{CL}$ 
accesorios, aumentando con ello la concentración de progesterona y la tasa de preñez (Rajamahendran y Sianangama 1992; Schmitt et al., 1996; Rendel, 1997; Rajamahendran et al., 1998; Santos et al., 2001).

El área y el diámetro de los CL en los tres grupos de aplicación hormonal se mantuvo constante en las vacas preñadas, pero tendiendo a disminuir en las vacas vacías tratadas con eCG (Figuras 1 y 2), y coincidiendo con el perfil de progesterona plasmática (Figura 3). Zahid y Cahit (2010) encontraron mayores niveles de progesterona y sin cambios considerables en el grupo tratado con GnRH más hCG. En forma similar, Cruz et al. (2009), trabajando con vacas de alta producción sometidas a estrés calórico consiguieron lecturas similares al presente estudio.

\section{Conclusiones}

El tratamiento con GnRH 14 días posIATF incrementa la tasa de preñez en vacas de alta producción que experimentaron estrés calórico.

\section{Literatura Citada}

1. Bartolome JA, Kamimura S, Silvestre F, Arteche AC, Trigg T, Thatcher $\boldsymbol{W W}$. 2006. The use of a deslorelin implant (GnRH agonist) during the late embryonic period to reduce pregnancy loss. Theriogenology 65: 1443-1453. doi: 10.1016/j.theriogenology.2005.08.017

2. Bartolome JA, Perez S, de la Sota RL, Thacher $W W .2012$. The effect of administering equine chorionic gonadotropin $(\mathrm{eCG})$ and human chorionic gonadotropin (hCG) post artificial insemination on fertility of lactating dairy cows. Theriogenology 78: 1110-1116. doi: 10.1016/j.theriogenology.2012.05.006

3. BonDurant RH. 2007. Selected diseases and conditions associated with bovine conceptus loss in the first trimester. Theriogenology 68: 461-473. doi: 10.1016/j.theriogenology.2007.04.022

4. Cruz JE, Elizondo CA, Ulloa AR. 2009. Efecto de la GnRH post inseminación sobre la concentración de progesterona y las tasas de concepción en vacas repetidoras Holstein en condiciones de estrés calórico. Tec Pecu Mex 47: 107-115

5. De Rensis F, Scaramuzzi RJ. 2003. Heat stress and seasonal effects on reproduction in the dairy cow - a review. Theriogenology 60: 1139-1151. doi: 10.1016/S0093-691X(03)00126-2

6. Diskin MG, Morris DG. 2008. Embryonic and early foetal losses in cattle and other ruminants. Reprod Domest Anim 43: 260-267. doi: 10.1111/j.14390531.2008.01171.x.

7. Ealy AD, Drost M, Hansen PJ. 1993. Developmental changes in embryonic resistance to adverse effects of maternal heat stress in cow. J Dairy Sci76: 2899-2905. doi: 10.3168/jds.S00220302(93)77629-8

8. García-Inspierto I, López-Gatius F. 2012. Effects of $\mathrm{GnRH}$ or progesterone treatment on day 5 post -AI on plasma progesterone, luteal blood flow and leucocyte counts during the luteal phase in dairy cows. Reprod Domest Anim 47: 224-229. doi: 10.1111/j.1439-0531.2011.01832. $\mathrm{x}$

9. Jordan ER. 2003. Effects of heat stress on reproduction. J Dairy Sci 86: 104-114. doi: 10.3168/jds.S0022-0302(03)74043-0

10. López-Gatius FP, Santolaria JL, Yaniz J, Rutllant M, López-Bejar. 2002. Factors affecting pregnancy loss from gestation Day 38 to 90 in lactating dairy cows from a single herd. Theriogenology 57: 1251-1261. doi: 10.1016/S0093-691X(01)00715-4

11. López-Gatius, F. 2003. Is fertility declining in dairy cattle? a retrospective study in northeastern Spain. Theriogenology 60: 89-99. doi: 10.1016/S0093691X(02)01359-6 


\section{López-Gatius F, Santolaria P, Martino} A, Delétang F, De Rensis F, 2006. The effects of GnRH treatment at the time of $\mathrm{AI}$ and 12 days later on reproductive performance of high producing dairy cows during the warm season in northeastern Spain. Theriogenology 65: 820-830. doi: 10.1016/j.theriogenology.2005.07.002

13. Narvaez J. 2010. Efecto de la administración de eCG entre los días 16 y 22 post inseminación artificial sobre la concepción y el retorno al celo en vacas lecheras. Tesis de Magíster. Córdoba, Argentina: Univ. Nacional de Córdoba. $18 \mathrm{p}$.

14. Núñez R. 2011. Utilización de gonadotrofina coriónica equina (eCG) en vacas de carne, sobre la tasa de preñez y pérdidas embrionarias en un programa de inseminación artificial a tiempo fijo. Tesis de Magíster. Córdoba, Argentina: Univ. Nacional de Córdoba. 56 p.

15. Rajamahendran R, Ambrose JD, Schmitt EJ, Thatcher MJ, Thatcher $W W .1998$. Effect of buserelin injection and deslorelin (GnRH-agonist) implants on plasma progesterone, $\mathrm{LH}$, accessory $\mathrm{CL}$ formation, follicle and corpus luteum dynamics in holstein cows. Theriogenology 50: 1141-1155. doi: 10.1016/s0093-691x(98)00215-5

16. Rajamahendran $R$, Sianangama $P C$. 1992. Effect of human chorionic gonadotrophin on dominant follicles in cows: formation of accessory corpora lutea, progesterone production and pregnancy rates. J Reprod Fertil 95: 577584. doi: 10.1530/jrf.0.0950577

17. Rendel A. 1997. Efecto de GnRH (Buseralina) en vacas de lechería, inyectadas al día 0 o 7 post inseminacion artificial. Tesis de Médico Veterinario. Valdivia, Chile: Univ. Austral de Chile. 54 p.

18. Santos J, Thatcher WW, Pool L, Overton MW. 2001. Effect of human chorionic gonadotropin on luteal function and reproductive performance of high- producing lactating Holstein dairy cows. J Anim Sci 79: 2881-2894. doi: 10.2527/ 2001.79112881x

19. Santos JE, Thatcher WW, Chebel RC, Cerri RL, Galvão KN. 2004. The effect of embryonic death rates in cattle in the efficacy of estrus synchronization programs. Anim Reprod Sci 82: 513-535. doi: 10.1016/j.anireprosci.2004.04.015

20. Schmitt EJ, Diaz T, Barros CM, de la Sota RL, Drost M, Fredriksson EW, Staples CR, et al. 1996. Differential response of the luteal phase and fertility in cattle following ovulation of the firstwade follicle with human chorionic gonadotropin or an agonist of gonadotropin-releasing hormone. J Anim Sci 74: 1074-1083. doi: 10.2527/1996.7451074x

21. Sreenan JM, Diskin MG, Morris DG. 2001. Embryo survival rate in cattle: a major limitation to the achievement of high fertility. In: Fertility in the high producing dairy cow. $26 \mathrm{Occ} \mathrm{Publ} \mathrm{Br} \mathrm{Soc}$ Anim Sci 26: 93-104. doi: 10.1017/ S0263967X00033619

22. Thatcher WW, Staples CR, DanetDesnoyers G, Oldick B, Schmitt EP. 1994. Embryo health and mortality in sheep and cattle. J Anim Sci 72(Suppl 3): 16-30. doi: 10.2527/1994.72suppl $316 \mathrm{x}$

23. Ullah G, Fuquay JW, Keawkhong T, Clark BL, Pogue DE, Murphey EJ. 1996. Effect of gonadotropin-releasing hormone at estrus on subsequent luteal function and fertility in lactating Holsteins during heat stress. J Dairy Sci 79: 19501953. doi: 10.3168/jds.S0022-0302(96)76565-7

24. Vanroose G, de Kruif A, Van Soom A. 2000. Embryonic mortality and embryopathogen interactions. Anim Reprod Sci 60: 131-143. doi: 10.1016/S03784320(00)00098-1

25. Wathes DC. 1992. Embryonic mortality and the uterine environment. J Endocrinol 134: 321-325. doi: 10.1530/jrf.0.0840393 
26. Willard S, Gandy S, Bowers S, Graves K, Elias A, Whisnant C. 2003. The effects of GnRH administration post insemination on serum concentrations of progesterone and pregnancy rates in dairy cattle exposed to mild summer heat stress. Theriogenology 59:
1799-1810. doi: 10.1016/s0093-691x(02)01232-3

27. Zahid P, Cahit K. 2010. The effects of GnRH and hCG used during and after artificial insemination on blood serum progesterone levels and pregnancy rate in cows. Kafkas Univ Vet Fak 16: 371-375. 